\title{
Common molecularcytogenetic alterations in tumors originating from the pineal region
}

\author{
FLORIAN BÖHRNSEN $^{1}$, CHRISTINA ENDERS ${ }^{2}$, HANS-CHRISTOPH LUDWIG ${ }^{3}$, \\ WOLFGANG BRÜCK ${ }^{4}$, LASZLO FÜZESI ${ }^{2}$ and ANGELIKA GUTENBERG ${ }^{3,5}$ \\ ${ }^{1}$ Clinic of Oral and Maxillofacial Surgery, ${ }^{2}$ Institute of Pathology, ${ }^{3}$ Department of Neurosurgery \\ and ${ }^{4}$ Institute of Neuropathology, Georg-August University Göttingen, Göttingen D-37075; \\ ${ }^{5}$ Department of Neurosurgery, Johannes Gutenberg University, Mainz D-76726, Germany
}

Received August 23, 2014; Accepted May 7, 2015

DOI: $10.3892 / \mathrm{ol} .2015 .3383$

\begin{abstract}
Tumors of the pineal region (PR) are rare and can be subdivided into four main histomorphological groups: Pineal-parenchymal tumors (PPT), germ cell tumors (GCT), glial tumors and miscellaneous tumors. The appropriate pathological classification and grading of these malignancies is essential for determining the clinical management and prognosis. However, an early diagnosis is often delayed due to unspecific clinical symptoms, and histological support is not always decisive to identify the diversity of tumors of the PR. The present study aimed to characterize 18 tumors of the PR using comparative genomic hybridization. All the tumors were primarily surgically resected without any previous irradiation or chemotherapy. In addition to chromosomal aberrations in PPT and different GCTs of the PR, the present study described, for the first time, the chromosomal changes in a few rare entities (solitary-fibrous and neuroendocrine tumors) of the PR. The tumors in the study, regardless of histology and World Health Organization grade, were characterized by frequent gains at 7, 9q, 12q, 16p, 17 and 22q, and losses at 13q. While the detection of chromosomal aberrations in these tumors appears not to be indicative enough of histological entities and their grade of malignancy, the present data may be of use to select genes of interest for higher resolution genomic analyses.
\end{abstract}

\section{Introduction}

Tumors of the pineal region (PR) are rare. The tumors are most commonly found in children and infants, while only $1 \%$ occur during adulthood $(1,2)$. The appropriate pathological

Correspondence to: Dr Florian Böhrnsen, Clinic of Oral and Maxillofacial Surgery, Georg-August University Göttingen, 40 Robert-Koch Street, Göttingen D-37075, Germany

E-mail: florian.boehrnsen@med.uni-goettingen.de

Key words: pineal region tumors, comparative genomic hybridization, pineoblastoma, pineocytoma, germ cell, chromosomal copy number aberrations classification and grading of the malignancy of tumors of the $\mathrm{PR}$ is essential for determining the clinical management and prognosis $(3,4)$. Tumors of the PR can be subdivided into four main histomorphological groups: Pineal-parenchymal tumors (PPT), germ cell tumors (GCT), glial tumors and miscellaneous tumors.

PPTs originating from the pineal gland itself account for 14-27\% of PR tumors (1,5-7). In addition to grade I pineocytoma and grade IV pineoblastoma, PPT of intermediate differentiation (PPTID) was recognized in the 2007 World Health Organization (WHO) classification as an intermediate-grade malignancy (II or III). Therefore, these tumors may have been summarized in the past under grade I pineocytoma or grade IV pineoblastoma (5). The origin of pineal cysts remains unclear, but they are believed to be non-neoplastic (8). GCTs are among the most abundant entities (40\%) in the PR, and include germinomas and non-germinomatous tumors, such as epidermoid cysts, teratomas and yolk sac tumors $(5,9,10)$. Glial tumors, most commonly grade I pilocytic astrocytomas, account for up to $25 \%$ of tumors of the PR $(5,11)$. Miscellaneous tumors of the PR include solitary fibrous tumors (SFT) (12) and metastases. Rarely, other brain tumors, including meningioma, glioblastoma, ependymoma, plexus papilloma and neuroendocrine tumors (NETs), can also occur in the PR (13-16).

The early diagnosis of tumors in the PR is often delayed through unspecific clinical symptoms. Imaging studies can be suggestive of the type of tumor of the PR, but only occasionally provide the exact diagnosis $(9,17,18)$. Serum and cerebrospinal fluid markers have been the most useful in the pre-operative evaluation, as $\alpha$-fetoprotein (AFP) and $\beta$-human chorionic gonadotropin $(\beta-\mathrm{HCG})$ are indicative of malignant GCT (19). Nevertheless, the absence of AFP or $\beta$-HCG does not rule out a malignant GCT (20). Markers of PPTs are not as well characterized as their germ cell counterparts, and include melatonin and the S-antigen, neither of which have proven valuable in establishing the correct diagnosis $(21,22)$. Additionally, the histopathological diagnosis of tumors of the PR is often difficult due to the inherently small size of the biopsies for diagnosis and the wide range of histological tumor entities in this brain region $(3,5)$. Immunohistochemical support is not always decisive to identify the histomorphological diversity of tumors of the PR $(1,11,23)$. 
Therefore, valuable diagnostic markers have to be defined to allow a histogenetically-based diagnosis. Recently, we described a chromosomal pattern, which can differentiate papillary tumors of the PR from other papillary tumors of the PR (24). Specific genetic changes in other tumors of the PR are yet to be determined. The present study aimed to characterize tumors of the PR using comparative genomic hybridization $(\mathrm{CGH})$. In addition to chromosomal aberrations in PPT and different GCTs of the PR, the present study describes, for the first time, chromosomal changes in a few rare entities of the PR.

\section{Materials and methods}

Patients. This study included 18 patients in whom tumors of the PR were primarily surgically resected without any previous irradiation or chemotherapy between 1997 and 2005. Inclusion criteria comprised follow-up data and successful cytogenetic analyses of the tumors. The study was performed with the patients' informed consent, and according to the guidelines and approval of the Local Ethics Committee (No. 12/11/10) of the Georg-August University Göttingen (Göttingen, Germany).

DNA preparation and $C G H$. The specimens were trimmed to ensure a minimum of $75 \%$ tumor cells in the sample. Tumor DNA was extracted from formalin-fixed and paraffin-embedded tumors of the PR by proteinase $\mathrm{K}$ digestion $(2 \mathrm{mg} / \mathrm{ml}$ final concentration; Roche Diagnostics $\mathrm{GmbH}$, Mannheim, Germany) followed by spin column purification (Qiagen GmbH, Hilden, Germany). The labeling of tumor DNA (nick translation) was performed with biotin-16-dUTP and normal reference DNA with digoxigenin-11-dUTP (both Roche Diagnostics $\mathrm{GmbH}$ ). The denatured DNA probe containing $2 \mu \mathrm{g}$ tumor DNA, $1.5 \mu \mathrm{g}$ reference DNA and $80 \mu \mathrm{g}$ COT-1 DNA was hybridized for 3 days to normal metaphase spreads on glass slides (15×15-mm cover glass area). The slides were then washed, blocked with bovine serum albumin solution and incubated with anti-biotin fluorescein-conjugated avidin (Vector Laboratories Inc., Burlingame, CA, USA) and rhodamine-conjugated anti-digoxigenin (Roche Diagnostics $\mathrm{GmbH}$ ) antibodies. Finally, the slides were washed and mounted in antifade solution (Vector Laboratories Inc.) containing $2.5 \mu \mathrm{g} / \mathrm{ml} \mathrm{4',6-diamidino-2-phenylindole} \mathrm{(DAPI)}$ counterstain.

Imaging and image analysis. Image acquisition was performed on a Zeiss Axioskop fluorescence microscope (Zeiss, Göttingen, Germany) equipped with three separate bandpass filters (a DAPI bandpass, a green single bandpass and a red single bandpass) and a high sensitivity monochrome charge coupled device camera (Photometrics, Tuscon, AZ, USA). For each analysis, the averaged chromosome-specific green-to-red fluorescence ratios and their $95 \%$ confidence intervals from at least 10 well-selected metaphases were plotted using the Quips CGH software (Applied Imaging, Newcastle, UK). A gain of DNA sequences was recorded at chromosomal regions where the hybridization resulted in a tumor-to-normal ratio of 1.2. Overrepresentations were considered amplifications when the fluorescence ratio values were $>1.5$ in a subregion of a chromosome arm. A loss of DNA sequences was recorded where the tumor-to-normal ratio was 0.8 . As an internal control, normal reference DNA was chosen from the opposite gender to ensure correct technical analyses. The chromosomal regions $1 \mathrm{p} 32$-pter, $13 \mathrm{p}, 14 \mathrm{p}, 15 \mathrm{p}, 19,21 \mathrm{p}$ and $22 \mathrm{p}$, and the known constitutive heterochromatic regions at 1q, 9q, 16q and Yq, and telomeric regions, were excluded from the analysis.

Statistical analysis. To evaluate chromosomal imbalance distribution in relation to diagnostic assignment, for each of the entities in the dataset, gain and loss frequencies were calculated. Copy number profiles were compared by generating a heatmap of gain and loss distributions. All statistical analyses are intended to be exploratory rather than confirmatory due to the relatively low number of patients per group. $\mathrm{P}<0.05$ was considered to indicate a statistically significant difference and were determined using the Student's t-test. Mean values and standard deviations are shown. Statistical analyses were performed using GraphPadPrism 5.0 software (GraphPad Software Inc., La Jolla, CA, USA).

\section{Results}

Clinicopathological data. The 18 tumors of the PR included 6PPTs, 1 pineal cyst, 6 pineal GCTs and 5 miscellaneous tumors (Table I; Fig. 1). Overall, $83.3 \%$ of the patients were male, with a mean age at the time of tumor diagnosis of $26.6 \pm 17.0$ years. The male patients were significantly younger at the time of diagnosis (22.8 \pm 3.7 years; range, 5 months -47 years) than the female patients $(45.4 \pm 10.9$ years; range, $24-60$ years; $\mathrm{P}<0.035)$.

\section{Comparative genomic hybridization}

PPTs. The analyzed pineal cyst revealed gain of $16 \mathrm{p}$ as a single chromosomal aberration. By contrast, one of the 2 grade II PPTIDs did not reveal any chromosomal aberrations. In the other grade II PPTID and in the 4 grade IV pineoblastomas, a mean of 8.2 net chromosomal aberrations (6.4 gains and 1.8 losses) was found. Gains at $12 q$ and $16 p$ were observed in 3 out of 5 tumors. Moreover, 2 out of 4 pineoblastomas of WHO grade IV demonstrated gains of $8 \mathrm{q}$ and whole chromosome 17, as well as loss of 13q (Table I).

Pineal GCTs. While the epidermoid cyst did not reveal any chromosomal aberrations, the mature teratoma and 4 germinomas analyzed showed a mean number of 12.8 net chromosomal changes, (8 gains and 4.8 losses). Common imbalances were gains of $12 q$ and loss of $13 q$ in 3 out of 4 germinomas. Additionally, 4 germinomas showed gains of 1q, 7, 16p, 17q and 22q, as well as losses of 9p. The mature teratoma resembled similar chromosomal aberrations observed in germinomas, also revealing gains of 1q, 7q, 16p, 17q and 22q, and losses of $9 \mathrm{p}$ (Table I).

Glial tumors of the PR. Grade I pilocytic astrocytomas revealed gains of 11q, 7q, 16p, 20q and 22q, as well as gains of whole chromosome 1 and 17 (Table I).

Miscellaneous tumors of the PR. Plexus papilloma was characterized by gains of $7 \mathrm{q}, 12,14 \mathrm{q}, 15 \mathrm{q}, 19 \mathrm{p}$ and $21 \mathrm{q}$. No chromosomal losses were observed. SFT of the PR showed gains on 7, 9q, 16p, 17 and 22q, and losses on 13q. The NET of the PR revealed the majority of cytogenetic net changes of all analyzed tumors of the PR, including gains on 5, 7, 8, 9, 


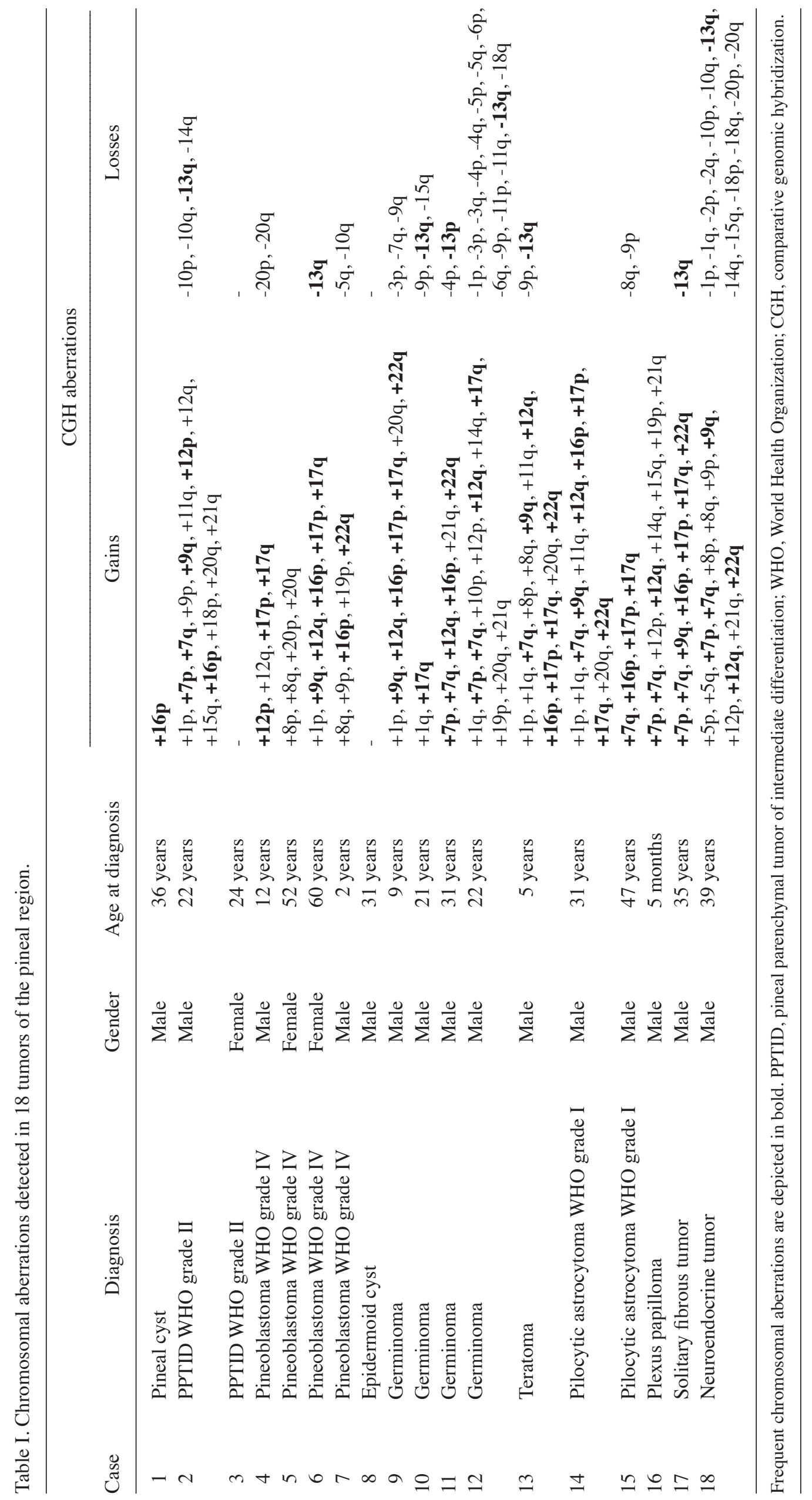




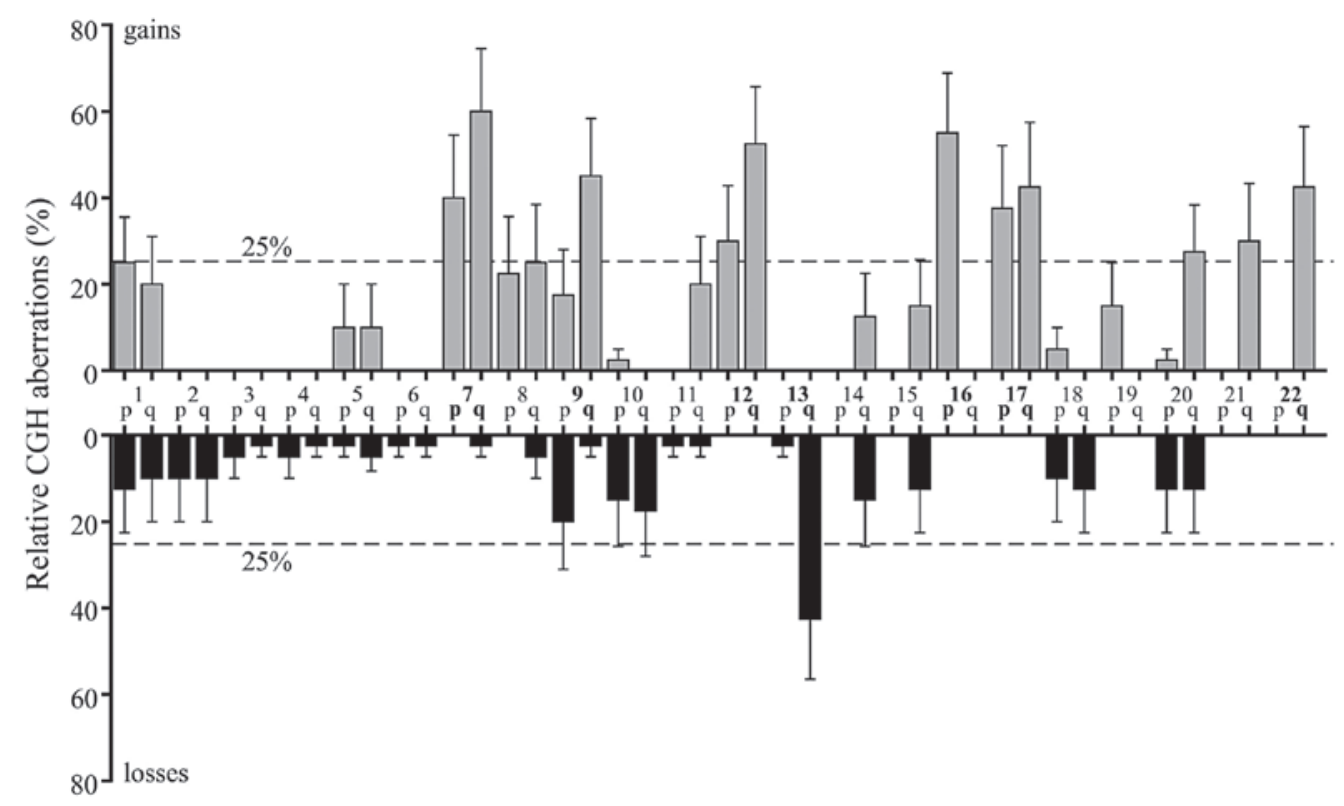

Figure 1. Chromosomal aberrations detected in 18 tumors of the pineal region. Error bars define the standard error of the mean. Frequent chromosomal aberrations are depicted in bold. CGH, comparative genomic hybridization.

$12 \mathrm{p}, 12 \mathrm{q}, 21 \mathrm{q}$ and $22 \mathrm{q}$, and losses on 1, 2, 10,13q, 14q, 15q, 18 and 20 (Table I).

\section{Discussion}

It can be expected that tumors originating from a single histological cell population will display the same cytogenetic pattern. However, classic concepts of tumorigenesis are incomplete with regard to explaining the diversity of heterogenic tumors of the PR (25). To date, the cytogenetic and molecular data on PR tumors have been sparse. The present study characterized 18 tumors of the PR and found no distinctive, but rather common cytogenetic aberrations for each tumor entity and origin.

Despite pineal cysts being considered as non-neoplastic lesions, $\mathrm{CGH}$ analyses in the present study revealed gains of 16p. The cytogenetic spectrum of grade II PPTID is limited, revealing gains of $4 \mathrm{q}$ and $12 \mathrm{q}$, and loss of chromosome 22 in two tumors each $(1,26)$. One of the grade II PPTIDs in the present study did not reveal any chromosomal imbalances, while the other grade II PPTID, in addition to gains of $16 \mathrm{p}$, showed a wide range of gains and losses. By using CGH (1) and array-CGH $(26,27)$, grade IV pineoblastomas show relatively low chromosomal rearrangements, with gains at $1,12 q$, $13 q$ and $19 p$, and losses at $13 q, 16 q$ and $22 q$ being the most frequent aberrations. In 2 out of 4 grade IV pineoblastomas in the present study, gains at $16 \mathrm{p}$ could also be observed. It may be concluded from the present results that gains at $16 \mathrm{p}$ appear to be the chromosomal hallmark of PPTs, but no correlation can be drawn between malignant progression and the number of molecular cytogenetic changes.

Cytogenetic analysis of cerebral GCT, particularly GCT of the PR, are seldom reported, with 27 pineal GCTs reported to date $(28,29)$. In these studies, the gain of $12 \mathrm{p}$, a hallmark in testicular GCT (30), was detected at varying frequencies. The most common gains at $1 \mathrm{q}, 8 \mathrm{q}, 12 \mathrm{p}$ and $17 \mathrm{q}$, as well as losses at $9 \mathrm{q}, 13 \mathrm{q}$ and $18 \mathrm{q}$, have been demonstrated in 27 GCTs of the PR, including the present study cases $(28,29)$. GCTs presented in the current study commonly displayed gains at 1q $(80 \%)$ and losses at $13 q(60 \%)$. Gains at 12 p and $8 \mathrm{q}$, and losses at $9 \mathrm{q}$ and $18 \mathrm{q}$ were observed in only $20 \%$ of cases.

Notably, only WHO grade I pilocytic astrocytoma was observed in the PR. Genetic analyses in grade I pilocytic astrocytomas of different regions are scarce. Moreover, pilocytic astrocytomas of different locations (hemispheric, cerebellar and chiasmatic) appear to show different biological characteristics with regard to biological aggressiveness (31). Gains have been described on chromosomes $1 \mathrm{p}$ (63\%), 2p (63\%), 9p (63\%), 9q (59.3\%), 16p (63\%), 17q, 19q $(55.5 \%)$ and $22(31,32)$. Losses have been shown for chromosomes 2p (40.7\%), 3 (11\%), 8p (63\%), 9q, 12q, 16p (77.8\%) and $16 \mathrm{q}(31,32)$. It is not possible to determine a correlation between these genetic alterations and the location of the tumors. The present results showed gains on chromosomes $1 \mathrm{p}, 9 \mathrm{q}$ and $16 \mathrm{p}$, as well as additional chromosomal changes. Losses were detected on chromosomes $8 \mathrm{q}$ and $9 \mathrm{p}$. Losses for $2 \mathrm{p}, 3,8 \mathrm{p}$ and $16 \mathrm{p}$ were not detected. These results emphasize the heterogeneity of grade I pilocytic astrocytomas I $(31,33)$. Among the miscellaneous tumors of the PR, a SFT, a plexus papilloma and an NET were characterized. The present study is the first cytogenetic characterization of a cerebral SFT occurring in the PR. Chromosomal gains were observed for 7, 9q, 16p, 17 and 22q, and loss at 13q. In 2010, the first study of a NET of the PR was published (15). Until then, NET of the PR had been unknown. The present study describes, for the first time, the cytogenetic alterations observed in a NET of the PR. The tumor demonstrated a high chromosomal instability with extended gains and losses. Frequent chromosomal changes have been demonstrated in NETs of the intestine, identifying several chromosomal clusters and tumor groups stressing the complexity and requirement for further $\mathrm{CGH}$ analysis of NETs of different locations (34). 
In summary, tumors of the PR in the present study, regardless of histology and $\mathrm{WHO}$ grade, were characterized by frequent gains at 7,9q, 12q, 16p, 17 and 22q. Gain of 16p appeared to be an common alteration in PPT. Gains at $12 \mathrm{q}$ were mainly observed in PPT and GCT. Additionally, losses to $13 \mathrm{q}$ were frequently observed in PPT and GCT, as well as in SFT and NET, but not in grade I pilocytic astrocytomas of the PR. Therefore, detection of chromosomal aberrations in these tumors may not be indicative of histological differentiation. However, the present data may of use for selecting genes of interest for higher resolution analyses like array-CGH, or for more recent analyses such as next generation sequencing (35).

\section{Acknowledgements}

Data shown in this publication are part of the doctoral thesis of Florian Böhrnsen.

\section{References}

1. Rickert CH, Simon R, Bergmann M, Dockhorn-Dworniczak B and Paulus W: Comparative genomic hybridization in pineal parenchymal tumors. Genes Chromosomes Cancer 30: 99-104, 2001.

2. Edwards MS, Hudgins RJ, Wilson CB, Levin VA and Wara WM: Pineal region tumors in children. J Neurosurg 68 : 689-697, 1988

3. Regis J, Bouillot P, Rouby-Volot F, Figarella-Branger D, Dufour H and Peragut JC: Pineal region tumors and the role of stereotactic biopsy: Review of the mortality, morbidity and diagnostic rates in 370 cases. Neurosurgery 39: 907-912, 1996.

4. Borja MJ, Plaza MJ, Altman N and Saigal G: Conventional and advanced MRI features of pediatric intracranial tumors: Supratentorial tumors. AJR Am J Roentgenol 200: W483-W503, 2013.

5. Louis DN, Ohgaki H, Wiestler OD, Cavenee WK, Burger PC, Jouvet A, Scheithauer BW and Kleihues P: The 2007 WHO classification of tumours of the central nervous system. Acta Neuropathol 114: 97-109, 2007.

6. DeGirolami U and Schmidek H: Clinicopathological study of 53 tumors of the pineal region. J Neurosurg 39: 455-462, 1973.

7. Kang JK, Jeun SS, Hong YK, Park CK, Son BC, Lee IW and Kim MC: Experience with pineal region tumors. Childs Nerv Syst 14: 63-68, 1998.

8. Al-Holou WN, Garton HJ, Muraszko KM, Ibrahim M and Maher CO: Prevalence of pineal cysts in children and young adults. Clinical article. J Neurosurg Pediatr 4: 230-236, 2009.

9. Smith AB, Rushing EJ and Smirniotopoulos JG: From the archives of the AFIP: Lesions of the pineal region: Radiologic-pathologic correlation. Radiographics 30: 2001-2020, 2010.

10. Pettorini BL, Al-Mahfoud R, Jenkinson MD, Avula S, Pizer B and Mallucci C: Surgical pathway and management of pineal region tumours in children. Childs Nerv Syst 2012.

11. Sajko T, Kudelic N, Lupret V, Lupret V Jr and Nola IA: Treatment of pineal region lesions: Our experience in 39 patients. Coll Antropol 33: 1259-1263, 2009.

12. Zhang J, Cheng H, Qiao Q, Zhang JS, Wang YM, Fu X and Li Q: Malignant solitary fibrous tumor arising from the pineal region: Case study and literature review. Neuropathology 30: 294-298, 2010.

13. Ozgural O, Kahilogullari G, Bozkurt M, Heper AO and Savas A: Primary pineal glioblastoma: A case report. Turk Neurosurg 23 572-574, 2013

14. Carson BS, Weingart JD, Guarnieri M and Fisher PG: Third ventricular choroid plexus papilloma with psychosis. Case report. J Neurosurg 87: 103-105, 1997.

15. Grozinsky-Glasberg S, Fichman S and Shimon I: Metastatic bronchial neuroendocrine tumor to the pineal gland: A unique manifestation of a rare disease. Hormones (Athens) 9: 87-91, 2010
16. Korogi Y, Takahashi M and Ushio Y: MRI of pineal region tumors. J Neurooncol 54: 251-261, 2001.

17. Dumrongpisutikul N, Intrapiromkul J and Yousem DM: Distinguishing between germinomas and pineal cell tumors on MR imaging. AJNR Am J Neuroradiol 33: 550-555, 2012.

18. Tosi MR, Ricci R, Bottura G and Tugnoli V: In vivo and in vitro nuclear magnetic resonance spectroscopy investigation of an intracranial mass. Oncol Rep 8: 1337-1339, 2001.

19. Legault G and Allen JC: Potential role of ventricular tumor markers in CNS germ cell tumors. Pediatr Blood Cancer 60: $1647-1650,2013$.

20. Seregni E, Massimino M, Nerini Molteni S, Pallotti F, van der Hiel B, Cefalo G, Spreafico F, Fossati F and Bombardieri E: Serum and cerebrospinal fluid human chorionic gonadotropin (hCG) and alpha-fetoprotein (AFP) in intracranial germ cell tumors. Int J Biol Markers 17: 112-118, 2002.

21. Leston J, Mottolese C, Champier J, Jouvet A, Brun J, Sindou M, Chazot G, Claustrat B and Fèvre-Montange M: Contribution of the daily melatonin profile to diagnosis of tumors of the pineal region. J Neurooncol 93: 387-394, 2009.

22. Korf HW, Gotz W, Herken R, Theuring F, Gruss P and Schachenmayr W: S-antigen and rod-opsin immunoreactions in midline brain neoplasms of transgenic mice: Similarities to pineal cell tumors and certain medulloblastomas in man. J Neuropathol Exp Neurol 49: 424-437, 1990.

23. Behari S, Jaiswal S, Nair P, Garg P and Jaiswal AK: Tumors of the posterior third ventricular region in pediatric patients: The Indian perspective and a review of literature. J Pediatr Neurosci 6: S56-71, 2011.

24. Gutenberg A, Brandis A, Hong B, Gunawan B, Enders C Schaefer IM, Burger R, Ostertag H, Gaab M, Krauss JK, et al: Common molecular cytogenetic pathway in papillary tumors of the pineal region (PTPR). Brain Pathol 21: 672-677, 2011.

25. Berger AH, Knudson AG and Pandolfi PP: A continuum model for tumour suppression. Nature 476: 163-169, 2011.

26. von Bueren AO, Gerss J, Hagel C, Cai H, Remke M, Hasselblatt M, Feuerstein BG, Pernet S, Delattre O, Korshunov A, et al: DNA copy number alterations in central primitive neuroectodermal tumors and tumors of the pineal region: An international individual patient data meta-analysis. J Neurooncol 109: 415-423, 2012.

27. Miller S, Rogers HA, Lyon P, Rand V, Adamowicz-Brice M, Clifford SC, Hayden JT, Dyer S, Pfister S, Korshunov A, et al: Genome-wide molecular characterization of central nervous system primitive neuroectodermal tumor and pineoblastoma. Neuro Oncol 13: 866-879, 2011.

28. Schneider DT, Zahn S, Sievers S, Alemazkour K, Reifenberger G, Wiestler OD, Calaminus G, Göbel U and Perlman EJ: Molecular genetic analysis of central nervous system germ cell tumors with comparative genomic hybridization. Mod Pathol 19: 864-873, 2006.

29. Rickert CH, Simon R, Bergmann M, Dockhorn-Dworniczak B and Paulus W: Comparative genomic hybridization in pineal germ cell tumors. J Neuropathol Exp Neurol 59: 815-821, 2000.

30. Sheikine Y, Genega E, Melamed J, Lee P, Reuter VE and Ye H: Molecular genetics of testicular germ cell tumors. Am J Cancer Res 2: 153-167, 2012

31. Belirgen M, Berrak SG, Ozdag H, Bozkurt SU, Eksioglu-Demiralp E and Ozek MM: Biologic tumor behavior in pilocytic astrocytomas. Childs Nerv Syst 28: 375-389, 2012.

32. Sanoudou D, Tingby O, Ferguson-Smith MA, Collins VP and Coleman N: Analysis of pilocytic astrocytoma by comparative genomic hybridization. Br J Cancer 82: 1218-1222, 2000.

33. Lambert SR, Witt H, Hovestadt V, Zucknick M, Kool M, Pearson DM, Korshunov A, Ryzhova M, Ichimura K, Jabado N, et al: Differential expression and methylation of brain developmental genes define location-specific subsets of pilocytic astrocytoma. Acta Neuropathol 126: 291-301, 2013.

34. Hashemi J, Fotouhi O, Sulaiman L, Kjellman M, Höög A, Zedenius $J$ and Larsson C: Copy number alterations in small intestinal neuroendocrine tumors determined by array comparative genomic hybridization. BMC Cancer 13: 505, 2013.

35. Wang Y, Makedon F and Pearlman J: Tumor classification based on DNA copy number aberrations determined using SNP arrays. Oncol Rep 15: 1057-1059, 2006. 